\title{
THE
}

\section{Localization and Diffusion in Quasi-2D Helium and Hydrogen Systems with Corrugated Boundaries}

\author{
A. E. Meyerovich \\ University of Rhode Island, sfo101@uri.edu
}

A. Stepaniants

University of Rhode Island

Follow this and additional works at: https://digitalcommons.uri.edu/phys_facpubs

Terms of Use

All rights reserved under copyright.

\section{Citation/Publisher Attribution}

Meyerovich, A. E., \& Stepaniants, A. (2000). Localization and Diffusion in quasi-2D helium and hydrogen systems with Corrugated Boundaries. Physica B: Condensed Matter., 284-288(1) , 234 -235. doi: 10.1016/ S0921-4526(99)02467-9

Available at: https://doi.org/10.1016/S0921-4526(99)02467-9

This Article is brought to you for free and open access by the Physics at DigitalCommons@URI. It has been accepted for inclusion in Physics Faculty Publications by an authorized administrator of DigitalCommons@URI. For more information, please contact digitalcommons-group@uri.edu. 


\title{
Localization and diffusion in quasi- $2 D$ helium and hydrogen systems with corrugated boundaries
}

\author{
Alexander E. Meyerovich ${ }^{1}$, Armen Stepaniants \\ Department of Physics, University of Rhode Island, Kingston, RI 02881, USA
}

\begin{abstract}
Localization length and diffusion coefficient are calculated for quantized quasi- $2 D$ low-temperature systems with randomly corrugated boundaries or substrates. Applications include electrons on helium or hydrogen surfaces, quasiparticles in capillaries and ultrathin films, surface states, ultracold neutrons in gravitational traps, etc.
\end{abstract}

Keywords: localization; transport; films; surface states

\section{Introduction: effective collision operator}

Scattering by random surface inhomogeneities is crucial in low-temperature systems of particles with large free paths. At first glance, the elastic surface scattering should not be much different from scattering by bulk impurities. However, while the impurity scattering is described in textbooks, a similar account of surface scattering is missing. Recently, we developed a simple transport theory for ballistic particles in ultrathin quantized systems with randomly corrugated walls [1].

Below we apply the results to quasi- $2 D$ helium and hydrogen systems in which the motion in $x$-direction perpendicular to the walls is quantized while the motion along the walls remains quasiclassical. This quantization replaces the spectrum $\epsilon(\mathbf{p})$ by a set of $2 D$ minibands $\epsilon_{j}(\mathbf{q})$ and results in the matrix form of the equations. The transition

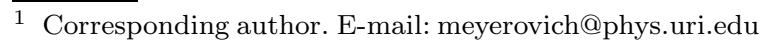

Preprint submitted to Physica $B$ probabilities between the states $(j, \mathbf{q}) \rightarrow\left(j^{\prime}, \mathbf{q}^{\prime}\right)$ caused by correlated collisions with walls are [1]

$$
W_{j j^{\prime}}=\frac{1}{4 m^{2}} \operatorname{Re}\left[\zeta \psi_{j}^{*(0) \prime} \psi_{j^{\prime}}^{(0) \prime} \psi_{j}^{(0)^{\prime}} \psi_{j^{\prime}}^{*(0) \prime}\right]
$$

where $\zeta\left(\mathbf{q}-\mathbf{q}^{\prime}\right)$ is the Fourier image of correlation function of surface inhomogeneities, $\psi_{j}^{(0) \prime}$ is the derivative of the wave function $\psi_{j}^{(0)}(x)$ on the wall for the same system but with uncorrugated, ideal walls.

The remaining issue is to calculate of $W_{j j^{\prime}}\left(\mathbf{q}, \mathbf{q}^{\prime}\right)$ for different system configurations and solve the transport equation with this $W_{j j^{\prime}}$. We did this analytically or semi-analytically for diverse localization and transport problems such as quasiparticles in ultrathin capillaries and films with rough walls, surface states on rough substrates, electrons on helium surface with ripplons, etc.

The matrix transport equation can be solved and the transport relaxation time operator $\widehat{\tau^{-1}}$ expressed via the angular harmonics ${ }^{(0,1)} W$ of 
the transition probability $W\left(\mathbf{q}-\mathbf{q}^{\prime}\right)$ in the same way as for the bulk impurities. The angular harmonics of the surface correlator $\zeta^{(0)}\left(\left|\mathbf{q}-\mathbf{q}^{\prime}\right|\right)-$ $\zeta^{(1)}\left(\left|\mathbf{q}-\mathbf{q}^{\prime}\right|\right)$ play the role of the impurity transport cross-section, $\sigma_{t r}\left(\left|\mathbf{q}-\mathbf{q}_{j j^{\prime}}\right|\right)=\sigma^{(0)}-\sigma^{(1)}$.

\section{Examples}

In three situations the transport equations for different minibands decouple from each other and the matrix $\tau^{-1}$ becomes diagonal, $\tau_{j j^{\prime}}^{-1} \simeq \tau_{j}^{-1} \delta_{j j^{\prime}}$ :

(i) only the first (lowest) quantum state $j$ is energetically accessible, and $\tau_{j j^{\prime}}^{-1}$ reduces to

$$
\frac{2}{\tau_{1}}=\sum_{\alpha, \beta}\left[{ }^{(0)} W_{11}^{(\alpha \beta)}-{ }^{(1)} W_{11}^{(\alpha \beta)}\right] \frac{q_{11}}{v_{11}}
$$

where $q_{j j^{\prime}}(\mathbf{q})$ is the solution of equation $\epsilon_{j}(\mathbf{q})=$ $\epsilon_{j^{\prime}}\left(\mathbf{q}_{j j^{\prime}}\right), v_{j j^{\prime}}$ is the corresponding velocity;

(ii) the clearance between the walls or the size of quantized state $L$ is smaller than the correlation radius $R$ of surface inhomogeneities making the interband transitions negligible in comparison to intraband scattering, $W_{j \neq j^{\prime}} \ll W_{j j}$ :

$$
\frac{1}{\tau_{j}}=\frac{1}{2} \sum_{\alpha, \beta}\left[{ }^{(0)} W_{j j}^{(\alpha \beta)}-{ }^{(1)} W_{j j}^{(\alpha \beta)}\right] \frac{q_{j j}}{v_{j j}} ;
$$

(iii) the particle wavelength is large, $q R \ll 1$, and all $W$ are constant with ${ }^{(0)} W=2 W(0)$, ${ }^{(1)} W=0$ (quantum reflection):

$$
\frac{1}{\tau_{j}}=\sum_{\alpha, \beta} \sum_{j^{\prime}} W_{j j^{\prime}}^{(\alpha \beta)}(0) \frac{q_{j j^{\prime}}}{v_{j j^{\prime}}} .
$$

In all other cases, the matrix transport equation has to be solved numerically.

The first example is the single-particle diffusion $D$ in quantized systems with rough walls at $L \ll R$,

$$
D=\frac{L^{2}}{4 \pi S \ell^{2} R^{2}} \sum_{j=1}^{S} \frac{v_{j}^{3} / j^{2} q_{j}\left(\partial \epsilon_{j \mathbf{q}_{j}} / \partial j\right)^{2}}{{ }_{1} F_{1}\left(\frac{3}{2}, 2,-2 q_{j}^{2} R^{2}\right)} .
$$

where $\ell$ is the amplitudes of the (Gaussian) correlation functions of surface inhomogeneities, and ${ }_{1} F_{1}$ is the hypergeometric function. This equation can be used for helium quasiparticles with an arbitrary energy spectrum $\epsilon(\mathbf{p})$.

Next, let us consider a particle near a corrugated wall in a holding field $e \mathcal{E} x$ such as electrons on helium surface or ultra-cold neutrons (with $m g x$ instead of $e \mathcal{E} x$ ) in the gravitational trap. The size of the first bound state is $L=(2 m e \mathcal{E})^{-1 / 3}$; the energy levels $\epsilon_{j} / e \mathcal{E} L$ are given by the zeroes of the Airy wave functions. In the longwave limit $R \sqrt{2 m E} \ll 1$

$$
D=\frac{2}{m^{4} g^{2} S^{2} \zeta(0)} \sum_{j=1}^{S}\left(E-\epsilon_{j}\right) \rightarrow \frac{8}{5} \frac{H L^{3}}{m S \zeta(0)}
$$

(the last equation is quasiclassical; $E$ is the particle energy, $H=E / e \mathcal{E}$ ).

The diffusion coefficient $D$ determines the mean free path $\mathcal{L}$ and the localization length $\mathcal{R}$ :

$$
\mathcal{R}(E)=\mathcal{L}(E) \exp \varphi, \varphi=\pi m S(E) D(E)
$$

where $S$ is the number of accessible minibands $\epsilon_{j \mathbf{q}}$. If only the first miniband is occupied,

$$
\varphi \equiv \pi m D=\frac{2 L^{6}}{R^{4} \ell^{2}} \frac{q^{2} R^{2}}{{ }_{1} F_{1}\left(\frac{3}{2} ; 2 ;-2 q^{2} R^{2}\right)} .
$$

For ultra-cold neutrons, the weak localization is feasible if the neutron velocities $v<2 \mathrm{~cm} / \mathrm{s}$ (at present, the velocities of trapped neutrons exceed $10 \mathrm{~cm} / \mathrm{s}$ ). It is more promising to observe localization of electrons on helium or hydrogen surfaces. One can also expect the localization of hydrogen particles bound to the surface of helium with ripplon corrugation (at $T \sim 1 K, R \sim 20 \AA, \ell \sim 0.8$ $\AA)$ when the coefficient in Eq.(2) is 0.3 , and the exponent $\varphi<12$ for particles with momenta $q R<2$. Similar parameters are expected for ${ }^{3} \mathrm{He}$ impurity surface states in superfluid ${ }^{4} \mathrm{He}$.

The work is supported by NSF grants DMR9705304 and INT-9724728. Detailed results will be published elsewhere.

\section{References}

[1] A.E. Meyerovich, and A. Stepaniants, Phys.Rev. B 58, 13242 (1998); Phys.Rev. B (1999) in print 